\title{
Correction to: Overcoming Antimicrobial Resistance of the Skin
}

Stephen K. Tyring, Stephen Andrew Moore, Angela Yen Moore, and Omar Lupi

\section{Correction to:}

S. K. Tyring et al. (eds.), Overcoming Antimicrobial Resistance of the Skin, Updates in Clinical Dermatology, https://doi.org/10.1007/978-3-030-68321-4

A revised manuscript for chapter 1 received from the editor and the same has been updated.

Production team inadvertently missed to fix the citations in chapter 2 and 12 . This has now been updated. 